\title{
The expression and modulation of CEACAM1 and tumor cell transformation
}

\author{
Valentina Fiori $^{(\mathrm{a})}$, Mauro Magnani ${ }^{(\mathrm{b})}$ and Maurizio Cianfriglia ${ }^{(\mathrm{c})}$ \\ (a) Diatheva srl, Fano (PU), Italy \\ ${ }^{(b)}$ Dipartimento di Scienze Biomolecolari, Università degli Studi di Urbino "Carlo Bo", Urbino (PU), Italy \\ (c) Dipartimento del Farmaco, Istituto Superiore di Sanità, Rome, Italy
}

\begin{abstract}
Background. In this review, we focus our discussion on one class of carcinoembryonic antigen-related cell adhesion molecules, Carcinoembryonic antigen-related cell adhesion molecules 1 (CEACAM1). This has been observed in several malignant transformations to be subjected to complex mechanisms of modulation and dysregulation. Aims. Restoration of CEACAM1 expression in tumor cell lines often abolishes their oncogenicity in vivo, and therefore, this adhesion molecule has been regarded as a tumor suppressor. In contrast, de novo expression of CEACAM1 is found with the progression of malignancy and metastatic spread in a large array of cancer tissues which include melanoma, Non Small Cell Lung Carcinoma (NSCLC) as well as bladder, prostate, thyroid, breast, colon and gastric carcinomas. Discussion. We report and discuss the most significant findings confirming at immunohistochemical and clinical level the correlation between poor prognosis and expression of CEACAM1 on the cell surface of tumors.
\end{abstract}

Key words: CEACAM1, cancer, dysregulation, NSCLC, melanoma, poor prognosis.

Riassunto (Espressione e modulazione cellulare di CEACAM1 e trasformazione tumorale). In questo articolo abbiamo focalizzato il nostro interesse su di una classe di molecole di adesione e più in particolare sul CEACAM1 che in diversi studi è stato osservato essere coinvolto nella trasformazione tumorale attraverso complessi meccanismi di modulazione e di alterata regolazione. Obiettivo. L'espressione di CEACAM1 risulta spesso associata con l'abolizione della oncogenicità di linee cellulari trasformate e pertanto questa molecola di adesione è stata considerata alla stregua di un immunosoppressore tumorale. Discussione. In contrasto con questa osservazione, in molti casi l'espressione de novo di CEACAM1 risulta essere associata con la progressione tumorale e la metastatizzazione di diverse forme di cancro che includono il melanoma, il carcinoma polmonare a non piccole cellule, il carcinoma della vescica, della prostata, mammario, tiroideo e del carcinoma gastrico e del colon. In questo articolo riportiamo i dati più significativi della letteratura corrente che dimostrano attraverso studi immunoistochimici e clinici una stretta correlazione fra prognosi infauste ed espressione del CEACAM1 sulla superficie di cellule tumorali.

Parole chiave: CEACAM1, cancro, alterata regolazione, NSCLC, melanoma, prognosi infausta.

\section{INTRODUCTION}

CEACAMs are cell surface glycoproteins involved in intercellular binding and belong to the immunoglobulin superfamily [1]. They are implicated in various cellular functions governing growth and differentiation and have an important role in insulin homeostasis [2], vascular neogenesis [3], and immune modulation [4]. Some CEACAM members (CEACAM1, CEACAM3, CEACAM5 and CEACAM6) have also been identified as receptors for host-specific viruses and bacteria in mice and humans, making these proteins an interesting example of pathogen/host coevolution [5-7]. Further, CEACAMs have been implicated in various intracellular signalling-mediated effects involved in the growth and differentiation of cancer- ous cells thus playing a key role in the modulation of various types of cancers.

In this review, we restrict our discussion to CEACAM1, which has been reported in several malignant transformations to be subjected to a complex mechanism of regulation.

CEACAM1 has the widest tissue distribution of all its family members. This receptor is found not only on different epithelial cells, but also on various leukocytes. Its expression can be induced in endothelial or $\mathrm{T}$ cells [8]. CEACAM1 shows intercellular homophilic interactions as well as heterophilic binding to CEACAM5 expressed on target cells $[9,10]$. In humans, 11 different CEACAM1 splice variants have been detected. CEACAM1 isoforms differ with 
respect to the number of extracellular immunoglobulin-like domains, membrane anchorage and the length of their cytoplasmic tail [4]. Isoforms with the long cytoplasmic domain, contain two tyrosine residues that can be phosphorylation and dephosphorylation targets during intracellular signalling and that constitute a functional immunoreceptor tyrosine-based inhibitory motif (ITIM) [11].

In some tumor cell lines, restoration of CEACAM1 expression abolishes their oncogenicity in vivo, thus this cell adhesion molecule has been regarded as a tumor suppressor [12]. In contrast, de novo expression of CEACAM1 actually increases the risk of metastasis in melanoma patients and is an independent predictor of survival in NSCLC patients [13, 14] raising the possibility that CEACAM1 expression might facilitate metastatic tumor spread. The aim of this investigation is firstly to analyze the association between the expression of CEACAM1 in lung cancer and melanoma and their metastatic deposits in lymph node and hematogenic sites. Secondly, we have also considered the transformation patterns of bladder, prostate, thyroid, breast and gastric cancer where, in parallel with CEACAM1 dysregulation, the progression of malignancy and metastatic spread may be observed. Recent insights into the role of CEACAM1 in cancer will be reported and discussed because of their importance in designing a more comprehensive role in cell transformation of this adhesion molecule as a feasible target for novel forms of in vivo immuno-therapeutic agents.

Multi-modal treatments that combine chemotherapy with surgery or radiotherapy have increased the survival rate of tumor patients. In particular, doseintensification has recently improved their cure rate, despite the increase of severe toxicity and high level of life-threatening late events, such as secondary malignancies.

The development of selective and better tolerated cancer therapeutics possibly represents an important goal in the research of new and more effective treatment of CEACAM1 positive tumors. Antibody-based cancer treatments have given promising results in several malignancies and specific monoclonal antibodies (mAbs) have demonstrated clinical efficacy in patients with solid tumors unresponsive to chemotherapy [15].

Although it seems unlikely to cure a large mass of tumors by the administration of mAbs alone [16], the combination of tumor targeting mAbs with conventional anticancer drugs represents an effective strategy to overcome the intrinsic or acquired resistance of solid tumors [17, 18] which often are extremely aggressive and show a low survival rate despite the adoption of multimodal treatments [19]. In order to identify a CEACAM1 tumor sub-population to which immunotherapeutic interventions can be applied via a specific antibody we have considered data obtained from already published results concerning biochemical, genetic and phenotypic aspects of CEACAM1 expressing human tumors. In particular, retrospective studies conducted on pa- tient tumor tissue specimens obtained from surgical resection have been extremely useful in determining the incidence and prevalence of this biomarker, and for generating hypotheses regarding the clinical relevance of CEACAM1 expression in the patient populations studied $[13,14]$. In the light of these studies we expected to notice CEACAM1 positive tumor subpopulations with less heterogeneous characteristics to which monoclonal antibody target therapy can be applied with an improved expectancy of efficacy. Our proposed study tries to respond at least in part to one of the critical aspects of personalized cancer therapy identifying a subgroup of patients who are most likely to benefit from a given therapy, given the presence of homogeneity in the expression and distribution of CEACAM1 in tumor cells.

\section{EXPRESSION OF CEACAM1 IN MALIGNANT MELANOMA AND ITS TUMOR PROGRESSION ROLE
IN THIS TUMOR ENTITY}

CEACAM1 plays a role in various physiological and pathophysiological human processes [8] and it has been suggested as a putative tumor suppressor, since its expression was found to be reduced in colon, endometrium, breast, and prostate cancer [20-24]. In contrast, a large body of evidence demonstrated that CEACAM1 is a marker for progression and metastatization of NSCLC and melanoma. In this regard, the various contributions of Thies and coworkers [13, 25-27] eliminate any controversy about the role of CEACAM1 in malignant melanoma. In one study published by Thies et al. on 2002 [13], CEACAM1 expression was immunohistochemically evaluated in 100 primary cutaneous malignant melanomas and correlated with metastasis in a 10year follow-up. In addition, CEACAM1 expression was analyzed in metastatic lesions (11 distant metastasis and 6 sentinel lymph node metastasis). The results obtained in this study showed a strong correlation between the expression of CEACAM1 in primary tumors and the subsequent development of metastatic disease.

In fact, among the 40 patients with CEACAM1positive primary melanomas 28 developed metastatic disease, compared with only 6 of 60 patients with CEACAM1-negative melanomas. In the majority of positive cases, the strongest CEACAM1 expression was observed at the invading front. In addition, CEACAM1 expression was preserved in the metastatic lesions. Kaplan-Meier analysis revealed a highly significant association between CEACAM1 expression and metastasis $(\mathrm{P}<0.0001)$; multivariate Cox regression analysis, including CEACAM1 expression status adjusted for standard prognostic indicators as tumor thickness, presence of ulceration, and mitotic rate, confirmed that CEACAM1 is an independent factor for the risk of metastasis with a predictive value superior to that of tumor thickness. 
These findings were confirmed and extended by Gamblicher and co-workers in a study published in 2009 [28] that analyzed if the dysregulation of CEACAM1 was associated with progression of malignant melanoma. In particular, the authors emphasized the lack of studies systematically investigating CEA (CEACAM5) and CEACAM1 expression in malignant melanoma and in its precursor lesions such as spreading melanoma (SSM), dysplastic nevi $(\mathrm{DN})$, and benign nevi (BN).

The data reported by Gamblicher et al., obtained from 106 cases of histopathologically proven $\mathrm{BN}$ $(\mathrm{n}=42), \mathrm{DN}(\mathrm{n}=22)$, thin SSM $(\mathrm{n}=21)$ and thick SSM $(n=21)$, revealed an apparently stepwise increase of CEACAM1 protein expression in melanocytic skin lesions at more advanced stages of neoplastic progression. CEACAM1 protein was significantly over expressed in thick SSM, thin SSM, and DN compared with BN. Moreover, CEACAM1 expression was significantly increased in thick SSM compared with DN with a staining intensity more pronounced at the invasive front of SSM. Similarly, they observed significantly increased CEA expression in DN and SSM compared with expression in BN. CEA expression in thick SSM was also increased compared with expression in DN. These data give support to previous studies indicating that CEACAM1 may have an important role in the development and progression of cutaneous melanoma.

An additional form of CEACAM1-associated biological resistance that renders the immune attack ineffective [29] has provided evidence in several reports by Markel et al. [30-32]. They showed that CEACAM1 expression is actively up-regulated in response to interferon-gamma on melanoma cells surviving specific lymphocyte-mediated aggression, conferring on these cells enhanced resistance against new attacks. Since effector functions of natural killer and $\mathrm{T}$ cells are inhibited by homophilic CEACAM1 interaction, immune escape could be responsible for the poor prognosis of CEACAM1 over-expressing tumors.

\section{THE IMPORTANCE OF MONOCLONAL ANTIBODIES SPECIFICITY FOR CEA (CEACAM5) AND CEACAM1 STAINING IN MELANOMA SAMPLES}

CEA staining with polyclonal or specific monoclonal antibodies (mAbs) is not rare in malignant melanoma. However, in independent studies conducted by Sanders et al. [33] and Ravindranath et al. [34] they found that anti CEA specific mAbs were not reactive with malignant melanoma. By contrast, Selby et al. [35] found CEA immunoreactivity with a polyclonal antibody in a significant number of malignant melanoma cases. However the authors did not exclude the possibility of a cross-reactivity of the polyclonal antibody with molecules such as nonspecific cross-reacting antigen (NCA; CEACAM6) that share antigenic sites with CEA. The data reported by Gamblicher [28] indicate that CEA protein detected by the anti-CEA mAb II-7 clone (DAKO, Glostrup, Denmark), is significantly over-expressed in melanocytic skin lesions with an increase of expression at more advanced stages of neoplastic progression. Hence, conflicting results for CEA detection in melanoma cells may be also explained by the specificity of the mAb used. In the same study, Gamblicher et al. showed that CEACAM1 protein detected by mAb 29H2 (catalog No. ab49510; Abcam, Cambridge, MA) is significantly over-expressed in thick SSM, thin SSM, and DN compared with $\mathrm{BN}$, with a stepwise over-expression in melanocytic skin lesions at more advanced stages of neoplastic progression. Thies et al. [13] suggested that $\mathrm{mAb} 4 \mathrm{D} 1 / \mathrm{C} 2$ specific for CEACAM1 is highly sensitive and may be a worthy complement to standard antibody panels for diagnosis of melanoma cells. They found that for the detection of CEACAM1, the monoclonal antibody 4D1/C2 was by far superior to the commercial CEACAM1 mAb 29H2, for detecting malignant melanoma cells. In particular, the sentinel lymph node sensitivity of 4D1/C2 was significantly higher ( $95 \% v s 40 \%)$. In addition, Thies and co-workers also showed that the sensitivity of the 4D1/C2 antibody against CEACAM1 is superior to the classic trio S-100, Melan-A, and HMB45 immunohistochemical markers for detecting melanoma cells.

\section{MELANOMA METASTASIS TO SENTINEL LYMPH NODES SELECTIVELY DENTIFIED BY CEACAM1 SPECIFIC MAB 4D1/C2}

Classification schemas for cancers are useful in predicting overall survival and the selection of patients for treatment. Historically, the most important factors in determining prognosis in patients with melanoma have been tumor thickness and lymph node status. In particular, sentinel lymph node (SLN) mapping offers greater accuracy in staging because it defines a subset of patients with microscopic metastatic disease and for this reason has become an integral part of the revised staging system for cutaneous malignant melanoma as proposed by American Joint Committee for Cancer [36, 37]. The histopathological assessment of SLN biopsy, reflecting the regional lymph node status is critically important for accurate staging [38], however, the best method to detect single melanoma cells within the SLN is still being controversially discussed. As SLNs may contain benign nevus cells inclusion (BNI), a significant diagnostic problem arises as BNIs can be mistakenly diagnosed as metastatic melanoma cells. Pathologic evaluation of SLNs using immunohistochemistry has improved diagnostic accuracy, yet there is no universally accepted standard protocol for pathologic processing of SLNs. For example, Karimipour and co-workers [39] evaluated the sensitivity and efficacy of S-100, HMB-45, and Melan-A stains for SLN biopsy. In this work, 99 
positive SLNs from 72 patients were retrospectively reviewed for the presence of microscopic metastatic melanoma on hematoxylin and eosin (H\&E), S-100, HMB-45, and Melan-A stained sections and sensitivities of each immunohistochemical stain were determined. The sensitivities of S-100, HMB-45, and Melan-A were $97 \%, 75 \%$, and $96 \%$ respectively. In the light of these results Karimipour et al. concluded that, given the lower sensitivity of mAb HMB-45, the practice for evaluation of SLN biopsy specimens in their group was modified using combinations of H\&E, S-100, and Melan-A without HMB-45. If the $\mathrm{H} \& \mathrm{E}$ sections were found negative or equivocal for metastatic melanoma, immunohistochemistry staining with S-100 and Melan-A was performed.

Kucher et al. [40] investigated whether the use of the newer marker Melan-A detected by a monoclonal antibody would improve the detection of metastatic melanoma in SLN biopsies and replace S-100 and HMB45 stains. In this study, 40 lymph nodes previously diagnosed with metastatic melanoma were retrospectively evaluated for S-100, HMB45, and Melan-A expression. In a parallel study, 42 SLN biopsies for metastatic melanoma detection were prospectively collected and evaluated for S100, HMB-45, and Melan-A expression. All lymph nodes with metastatic melanoma from the retrospective study demonstrated S-100 reactivity, five failed to express either HMB-45 or Melan-A, and one of the metastasis tested positive for S-100 and HMB45 but failed to show reactivity with Melan-A. In the prospective study 10 lymph nodes from 42 cases positive for metastatic melanoma, were positive for S-100, 9 were positive for HMB-45, and 9 were positive for Melan-A. Then, the authors concluded that Melan-A, although very specific, cannot replace the use of S-100 and HMB-45 for the detection of metastatic melanoma in SLNs. It can, however, substitute HMB-45 with equally good results.

Hence, micrometastasis in a lymph node can be easily missed on routine H\&E-stained sections, therefore, S-100 HMB-45 and Melan-A immunohistochemical stains are standardly performed on grossly negative SLNs for detection of metastatic melanoma. Each of these immunohistochemical markers, however, is not ideal.

Thies and co-workers criticize the specificity of reagent used for melanoma detection in SLN and propose other immunological and molecular methods. In a detailed overview [25] they reported that S100 shows frequent background staining of Langerhans cells, dendritic cells, macrophages and Schwann cells. MelanA stains normal melanocytes as well as malignant melanoma cells. HMB45 being a marker of melanocytic differentiation is more specific but less sensitive. Detection of mRNA from melanocyte-associated genes such as tyrosinase and MART in SLNs by reverse transcriptionpolymerase chain reaction (RT-PCR) is a sensitive indicator for the presence of melanoma or nevus cells, but again it does not enable the distinction be- tween both, thus the true incidence of metastatic melanoma cells might be overestimated. Therefore, it is urgent to identify tumor markers suitable for highly sensitive and specific analyses of melanoma cells within SLNs.

A growing body of evidence indicates that alterations of adhesion molecules play a pivotal role in the development of recurrent, invasive and distant metastasis. Loss of intercellular adhesion represents the first step in the process that leads malignant cells to escape from their site of origin and finally invade and metastasize. In addition to participating in tumor invasiveness and metastasis, adhesion molecules regulate or significantly contribute to a variety of functions including signal transduction, cell growth, differentiation, site-specific gene expression, morphogenesis, immunologic function, cell motility, wound healing, and inflammation [41].

During tumor metastasis, modulation of cell adhesion molecules expression and aberrant glycosylation of cell membrane molecules can be analyzed using lectins. In order to define the glycoconjugated profiling of primary melanoma and the presence of genuine melanoma tumor cells in its SLN and distant metastasis, Thies et al. [25] analyzed the expression of the two adhesion molecules, CEACAM1 and L1, using specific antibodies (L1-11A against L1 and 4D1/C2 against CEACAM1), in comparison with standard immunological probes which include Melan A, S100 and HMB45. To complete the above mentioned study, Thies and co-workers used a panel of tumor binding lectins such as HPA, the lectin from Helix pomatia which recognizes terminal alpha N-acetylgalactosamine residue, the lectin ML1 from mistletoe and peanut lectin PNA. Previous histochemical studies have demonstrated that expression of HPA-binding glycoproteins by cancer cells is a marker of metastatic competence and poor prognosis in a range of common human adenocarcinomas, including those of breast, stomach, ovary, esophagus, colorectum, thyroid and prostate [42]. Mistletoe lectin ML-1 and peanut lectin PNA binding sites are widespread in human tissues, with staining patterns varying by tissue type. The lectin ML-1 seems to induce an anti-angiogenic response in the host suggesting that the anti-metastatic effect, observed on a series of tumor cell lines in mice, is in part due to an inhibition of tumor-induced angiogenesis and in part due to an induction of apoptosis [43]. PNA is selective for acrosomes in rat and human sperm, $[44,45]$ and has also been used to label the synaptic extracellular matrix in the study of developing neuromuscular junctions [46] and serves as a marker for certain melanomas [47, 48].

Thies et al. assessed the expression of L1, CEACAM1, and binding of the lectins HPA, ML-I and PNA, in benign nevi $(n=12)$, primary melanomas (PTs: $n=$ 67), their corresponding sentinel lymph nodes (SLNs: $\mathrm{n}=40$ ), and distant metastasis (DMs: $\mathrm{n}=35$ ). In this study they found that sensitivity and specificity of anti CEACAM1 mAb 4D1/C2 and L1 exceeded 


\begin{tabular}{|c|c|c|c|c|}
\hline Tumor type & $\begin{array}{l}\text { Hystological aspect of } \\
\text { CEACAM1 expression }\end{array}$ & Clinical relevance & Remarks & References \\
\hline Melanoma & $\begin{array}{l}\text { *BN 0-3**; DN, 0-10; Thin } \\
\text { SSM, 2-20; Thick SSM, } \\
\text { 10-80. }\end{array}$ & $\begin{array}{l}\text { Correlation with tumor } \\
\text { progression and poor survival }\end{array}$ & $\begin{array}{l}\text { Normal skin cells adjacent to the } \\
\text { tumor lesion did not show } \\
\text { CEACAM1 expression }\end{array}$ & $\begin{array}{l}\text { Gambichler } \\
\text { et al., } 2009 \text { [28] }\end{array}$ \\
\hline $\begin{array}{l}\text { Non-small-cell } \\
\text { lung cancer } \\
\text { (NSCLC) }\end{array}$ & $\begin{array}{l}\text { CEACAM1 expression in } \\
82 / 82 \text { NSCLC specimen; } \\
\text { increased MVD }{ }^{\star \star \star} \text { in the } \\
\text { vicinicity of tumors }\end{array}$ & $\begin{array}{l}\text { MVD expression represents } \\
\text { an indipendent prognosticator } \\
\text { for unfavorable cancer related } \\
\text { survival }\end{array}$ & $\begin{array}{l}\text { Normal lung cells adjacent to the } \\
\text { tumor lesion did not show } \\
\text { CEACAM1 expression }\end{array}$ & $\begin{array}{l}\text { Dango et al., } \\
2008 \text { [56] }\end{array}$ \\
\hline Colon carcinoma & $\begin{array}{l}\text { IH analysis of CEACAM-S } \\
\text { and CEACAM-L showed } \\
\text { staining at the invasion } \\
\text { front of tumor }\end{array}$ & $\begin{array}{l}\text { Multivariate analysis shows that } \\
\text { CEACAM-L dominance was and } \\
\text { independent risk factor for lymph } \\
\text { node metastasis. }\end{array}$ & $\begin{array}{l}\text { CEACAM-L dominance is associated } \\
\text { with short survival and is important } \\
\text { for colorectal cancer cell invasion } \\
\text { and migration }\end{array}$ & $\begin{array}{l}\text { leda et al., } \\
2011 \text { [72] }\end{array}$ \\
\hline $\begin{array}{l}\text { Urinary Bladder } \\
\text { Cancer }\end{array}$ & $\begin{array}{l}\text { CEACAM1 immunostaining } \\
\text { is absent in normal } \\
\text { urothelium but appears } \\
\text { in endothelial cells of UCB }\end{array}$ & $\begin{array}{l}\text { Higher CEACAM1 expression } \\
\text { levels are associated with } \\
\text { cancer presence and muscle } \\
\text { invasive cancer }\end{array}$ & $\begin{array}{l}\text { High CEACAM1 is detect in urinary } \\
\text { UCB ( } 207 \mathrm{ng} / \mathrm{ml} \text { versus } 0 \mathrm{ng} / \mathrm{ml}\end{array}$ & $\begin{array}{l}\text { Oliveira-Ferrer } \\
\text { et al., [54] }\end{array}$ \\
\hline Thyroid carcinoma & $\begin{array}{l}\text { CEACAM1 is not appreciably } \\
\text { expressed in normal thyroid } \\
\text { tissues }\end{array}$ & $\begin{array}{l}\text { CEACAM1 is expressed in } \\
\text { thyroid carcinoma cell line } \\
\text { derived from tumors that show } \\
\text { aggressive behavior }\end{array}$ & $\begin{array}{l}\text { CEACAM1 reactivity was associated } \\
\text { with metastatic spread but not } \\
\text { with increased tumor size }\end{array}$ & $\begin{array}{l}\text { Liu et al., } \\
2007 \text { [57] }\end{array}$ \\
\hline Gastric carcinoma & $\begin{array}{l}\text { All tissue samples of gastric } \\
\text { carcinoma examined from } \\
2001 \text { to } 2006 \text { expressed } \\
\text { CEACAM1 with membranous } \\
\text { or cytoplasmic staining }\end{array}$ & $\begin{array}{l}\text { A more malignant } \\
\text { transformation in } \\
\text { gastric carcinoma is associated } \\
\text { with higher cytoplasmic } \\
\text { staining }\end{array}$ & $\begin{array}{l}\text { The transformation of CEACAM1 } \\
\text { distribution from membrane to } \\
\text { cytoplasm is an important incident for } \\
\text { the reverse effects on the tumorous } \\
\text { angiogenesis facilitating metastasis of } \\
\text { carcinoma cells to lymph nodes }\end{array}$ & $\begin{array}{l}\text { Zhou et al., } \\
2009[63]\end{array}$ \\
\hline
\end{tabular}

that of the standard markers MelanA, S100, and HMB45 in single marker use so these antibodies could be a worthy completion to standard antibody panels for diagnosis of melanoma cells. Moreover, both molecules seem to be functionally involved in lymphatic and haematogenous spread, and are thus promising targets for immunotherapy. Lectin binding was found in PTs and DMs (HPA: 69\% and $77 \%$; ML-I: $82 \%$ and $77 \%$, respectively), but rarely in SLNMs (HPA: 20\%, ML-I: 20\%, PNA: 5\%, respectively). The authors concluded that CEACAM1 and L1 seem to have multidimensional importance for diagnosis, prognostic estimations and treatment of malignant melanoma. As they are expressed in the overwhelming majority of lymphatic and hematogenous metastasis, they represent a potential target for antimetastatic immunotherapy.

\section{THE ROLE OF CEACAM1 IN CANCER}

The role of CEACAM1 in tumor cells is still a matter of active investigation for the putative, apparently contradictory, function of down- and upregulation in different types of cancer. Several studies have challenged the previously postulated con- cept of a tumor suppressive effect of CEACAM1 since its putative angiogenic function.

Tumor growth and progression depend on angiogenesis, which is regulated by angiogenic activators and inhibitors [49, 50]. In the intricate angiogenesis system, cell adhesion molecules play an important role in vascular morphogenesis and endothelial signaling [51]. Human CEACAM1 is an adhesion molecule with pro angiogenic activity acting as a major effector of vascular endothelial growth factor (VEGF). It has been shown that the angiogenic effects of CEACAM1 are additional to those of the VEGF: the expression of CEACAM1 was up-regulated by VEGF, and VEGF-induced in vitro tube formation was completely blocked by a monoclonal anti-CEACAM1 antibody [52, 53].

Oliveira-Ferrer and co-workers [54] have observed that CEACAM1, which is ubiquitously expressed in the luminal surface of normal bladder urothelium, is down regulated in bladder cancer cells while it is concurrently up regulated in endothelial cells of adjacent blood vessels. This differential switch in CEACAM1 expression is accompanied by an up-regulation of pro-angiogenic and pro-lymphangiogenic factors such as VEGF-C and -D. Interestingly, the 
up-regulation of CEACAM1 during this angiogenic activation of endothelial cells is detectable both in a membrane-bound form and in the supernatant of these cells [52]. So, strategies targeting endothelial CEACAM1 may be of benefit for anti-angiogenic bladder cancer therapy. Similar observations were made by Tilki et al. [55] in prostate cancer where epithelial CEACAM1 downregulation in prostate intraepithelial neoplasia (PIN) is accompanied by upregulation of VEGF-A, -C and -D and concurrently, CEACAM1 is upregulated in adjacent blood vessels. In electron microscope studies conducted by the authors, the majority of PIN-associated blood vessels were structurally destabilized exhibiting endothelial fenestration, trans- and inter-endothelial gaps and basement membrane degradation, indicating activation of angiogenesis. In some PIN areas, invasion of single tumor cells into the destabilized blood vessels was observed. Based on these data, the authors concluded that strategies to either conserve the epithelial CEACAM1 or to target endothelial CEACAM1 might be useful for an anti-angiogenic therapy of prostate cancer.

In order to investigate a possible angiogenic influence of CEACAM1 in non-small-cell lung cancer (NSCLC), Dango et al. [56] analyzed specimens from 82 consecutive patients with completely resected NSCLC, examining microvessel density (MVD) and CEACAM1 expression, immunohistochemically stained using the monoclonal anti-CD31 antibody JC70A and monoclonal anti -CEACAM-1 antibody $4 \mathrm{D} 1 / \mathrm{C} 2$ respectively. The authors observed that increased CEACAM1 expression in the tumor cells and increased MVD in the vicinity of the primary tumors in NSCLC correlate with the development of distant metastasis and decreased cancer-related survival. In the cited article, the authors demonstrated that the prognostic impact of CEACAM1 depends also on the prognostic influence of MVD while MVD itself represents an independent prognosticator for unfavorable cancer related survival. This led to the suggestion that increased CEACAM1 expression in the tumor cells stimulates angiogenesis. In this study Dango et al., showed that CEACAM1 has an important role in the development and progression of cancer. In addition, they did not agree with the postulated role of CEACAM1 being a tumor suppressor which is based on the observations of down regulation of CEACAM1 in a number of different cancers and the inhibition of in vivo tumor growth upon re-expression of CEACAM1 in tumor cells.

In another study, Liu and co-workers [57] demonstrated that CEACAM1 is not appreciably expressed in normal thyroid tissue or benign thyroid tumors while it is expressed in thyroid carcinoma cell lines derived from tumors that exhibit aggressive behavior. In a human thyroid tissue array, CEACAM1 reactivity was associated with metastatic spread but not with increased tumor size. These findings identify CEACAM1 as a unique mediator that restricts tumor growth whereas increasing metastatic poten- tial providing a putative mechanism underlying the spectrum of biologic with thyroid cancer.

Therefore, detailed investigations of the expression patterns of CEACAM1 in many different types of cancer are crucial in determining how CEACAM1 might be involved in carcinogenesis and what significance the altered CEACAM1 expression might have for diagnosis, prognosis, and treatment of distinct cancer types. The emerging picture of the involvement of CEACAM1 is thus very complex. Nonetheless, the CEACAM1 expression pattern has been confirmed, in independent laboratories, to be a useful and valuable prognosticator parameter in different types of tumors.

\section{EXPRESSION AND ASSOCIATION OF INHIBITOR OF DIFFERENTIATION (ID-1) AND CEACAM1 IN BENIGN, PREMALIGNANT, AND MALIGNANT LESIONS OF HUMAN MAMMARY GLANDS}

Breast carcinogenesis is probably a multistep process that evolves from a benign stage at the terminal duct lobular unit (TDLU), progresses through an intermediate proliferative stage with or without the atypical hyperplastic epithelium, and eventually terminates in situ and invasive ductal carcinoma [58].

The inhibitor of differentiation/DNA synthesis (Id-1) protein functions mainly as a dominant inhibitor of the bHLH transcription factor and plays a critical role in regulating cell proliferation [59]. It may be involved in the malignant progression of human cancer. In particular, Id-1 is constitutively expressed in highly aggressive and invasive human breast cancer cells [60] and its expression is related with poor prognosis in some cases [61].

In a recent work, Liu and co-workers [62] analyzed the expression of Id-1 and CEACAM1 proteins by immunohistochemistry in 97 cases of benign, premalignant, and malignant lesions of human mammary glands. They showed that, in benign lesions, Id-1 expression is down-regulated and CEACAM1 is expressed with an apical membrane pattern. In contrast, in premalignant and malignant lesions, CEACAM1 is expressed with cytoplasmic and uniform membranous patterns and correlated with Id1 protein over-expression. These observations could indicate that Id-1 over-expression may inhibit normal apical expression and promote the movement of CEACAM1 from apical membrane to bilateral membrane and cytoplasm, and this transformation may occur in the early stage of the tumorigenesis. In this process, Id-1 should be regarded as a transforming factor for CEACAM1 expression and movement but the molecular basis of the transformation remains unknown.

Similar changes on CEACAM1 cellular distribution were also observed by Zhou et al. [63] in gastric carcinoma. The authors analyzed CEACAM1 expression in paraffin wax sections of 222 patients with gastric adenocarcinomas classified into three histo- 
types following the Laurén classification: intestinal, diffuse, and mixed carcinomas. All of the collected gastric carcinomas expressed CEACAM1 with cytoplasmic or membranous staining. CEACAM1 was expressed mainly with a membranous pattern in the intestinal carcinomas, and with a cytoplasmic pattern in the diffuse carcinomas that generally carry a worse prognosis. Therefore, membranous distribution of CEACAM1 changing to cytoplasmic staining may indicate a more malignant transformation in gastric carcinomas. Moreover, in this work the authors observed high MVD more frequently in the tumors with membranous expression, and low MVD in the tumors with cytoplasmic staining ( $\mathrm{P}$ $<0.0001)$. So, they concluded that membranous CEACAM1 may promote angiogenesis in the carcinoma areas while cytoplasmic CEACAM1 may inhibit angiogenesis. Finally, consistent with the observations on other types of cancer, the authors found that the patients with lymph node metastasis showed high expression of CEACAM1 in respect to patients without lymph node metastasis that showed low expression. These data also confirm the involvement of CEACAM1 in the development of cancer metastasis in gastric carcinoma.

\section{CEACAM1 AND LUNG CANCER}

The tumor-suppressive role of CEACAM1 is still a matter of active discussion and not universally accepted by considering (1) the various and complex physiological roles in which cell adhesion molecules are involved in tumorigenesis and (2) the observed linkage between CEACAM1 expression level and malignancy in tumor tissues such as lung cancer and melanoma.

As reported above, cell adhesion molecules play a key role in tumor invasion and metastasis [64]. The loss of cell-cell binding that closely correlates with differentiation and the invasive potential of malignant tumors is accompanied by a loss of, or alteration, in expression of cell adhesion molecules [65].

It is an exciting issue to address why a cell adhesion molecule is able to suppress tumor growth yet promote tumor progression. Although the mechanism of CEACAM1 suppressive action is largely unresolved, several reports suggested that it depends on the presence of its cytoplasmic domain that is involved in signal transduction interaction [66]. In tumor progression CEACAM1 exhibits properties of an angiogenic factor in endothelial cells and acts as a major effector of VEGF, suggesting that CEACAM1 expression might promote metastasis by the induction of angiogenesis at the metastatic site.

Lung cancer is the leading cause of cancer-related mortality in Europe and the United States [67]. NSCLC accounts for approximately $80 \%$ of all lung cancer patients. Most lung carcinomas are diagnosed at an advanced stage, conferring a poor prognosis. The standard treatment for early-stage NSCLC primarily involves surgery, chemotherapy, or radiation therapy. However, it results in a 5-year survival of only $50-60 \%$ patients in stage I and II [68]. Moreover, patients with the same stage of disease have markedly different rates of disease progression. There's an urgent need for better prognostic parameters in operable NSCLC for the identification of subgroups that could benefit from adjuvant therapy after surgical resection.

In order to elucidate the role of CEACAM1 in the progression and survival of patients with operable non-small cell lung cancer (NSCLC), Sienel et al. [69] analyzed tumor samples from 145 consecutive patients with completely resected NSCLC by assessing CEACAM1 expression immunohistochemically detected by the mAb 4D1/C2. The authors found a significant association between CEACAM1 expression and status of the tumors. In all patients sections of normal bronchiolar epithelium exhibited no immunostaining. In contrast, 73 tumors (50.4\%) showed between 1 and 66 CEACAM1-positive tumor cells and 72 tumors (49.6\%) exhibited even a higher percentage of positive cells. Sienel and coworkers, following a detailed statistical analysis, concluded that the absence of CEACAM1 in normal lung tissue and its expression in tumor cells argues against a tumor-suppressive role of CEACAM1 in NSCLC. Rather, elevated CEACAM1 expression was associated with severe disease progression and an unfavorable prognosis with a tendency to reduced cancer-related survival in the total population and a significant association to unfavorable outcomes for advanced diseases. A multivariate Cox regression analysis of the total population confirmed that CEACAM1 expression was an independent predictor for reduced cancer-related survival. The predictive power of CEACAM1 expression was highlighted by including known prognostic factors such as pT status, $\mathrm{pN}$ status, or patient age into the multivariate analysis. In this analysis, only CEACAM1 expression and $\mathrm{pN}$ status remained as independent predictors for cancer-related survival.

The study conducted by Thies et al. [27] on malignant melanoma patients has challenged the concept of any tumor-suppressive effect of CEACAM1 and reinforced the role of tumor induction and progression of this cell adhesion molecule. For the evaluation of CEACAM1 expression in melanoma the same $\mathrm{mAb} 4 \mathrm{D} 1 / \mathrm{C} 2$ recognized by Sienel et al. as a high specific for CEACAM1 detection in lung cancer was utilized.

Because CEACAM1 expression had a significant clinical impact in advanced tumor stages, the inhibition of this molecule may be a new therapeutic strategy for patients with advanced operable NSCLC.

\section{EXPRESSION OF CEACAM1 IN ADENOCARCINOMA OF THE LUNG}

Histologically, NSCLCs can be subdivided into adenocarcinomas, squamous cell carcinomas, and large-cell carcinomas. Adenocarcinomas have been 
increasing in incidence during the past decades and have become the most common type of NSCLC in the United States and in Western Europe [70].

To date, no satisfactory prognostic markers for the stratification of lung adenocarcinomas has emerged beyond the classical tumor-node-metastasis classification which rely on the anatomic description of the tumor spread without considering any biological insight into the mechanisms leading to the metastatic potential. However, as discussed above, cell-to-cell and cell-to-matrix interactions play an important role in this process, and several cell adhesion molecules mediating these interactions are involved in the metastatic spread of tumors.

The prognostic relevance of CEACAM1 expression in adenocarcinomas has been investigated by Laack et al. [14] in order to possibly identify a new prognostic marker in this clinically important tumor entity. The expression of CEACAM1's main carbohydrate determinant, sialyl Lewis X, was also investigated by this group, as it has been shown to be a prognostic marker in its own right and may in addition be functionally involved in CEACAM1mediated adhesion. In this study, sections from 93 patients with adenocarcinomas of the lung were immunohistochemically investigated using monoclonal anti-CEACAM1 and sialyl Lewis X antibodies. Sixty-one tumors were classified as CEACAM1positive, and 32 were classified as CEACAM1-negative. Patients with CEACAM1-positive tumors had a significantly poorer overall $(\mathrm{P}=0.00025)$ and relapse-free $(P=0.00029)$ survival than those with CEACAM1-negative tumors. Only 3 patients did not express the sialyl Lewis $\mathrm{X}$ glycotope, whereas 90 tumors (97\%) were sialyl Lewis X-positive. In multivariate Cox regression analysis, next to tumor stage and sex, only the expression of CEACAM1 was a significant independent prognostic factor for survival. It is interesting to note that CEACAM1 is not expressed in the normal bronchial or alveolar epithelium. Laack et al., concluded that since the expression of CEACAM1 is an independent prognostic factor in resectable adenocarcinomas of the lung, it can be used to stratify patients into low-risk and high-risk groups. In contrast, the expression of sialyl Lewis $\mathrm{X}$ is of no prognostic relevance because it was expressed in $97 \%$ of all investigated tumors, and most likely has no influence on the function of CEACAM1 in this tumor entity.

In a more recent study, Thöm et al. [71] analyzed CEACAM1 expression in primary tumors, lymph nodes and hematogenous metastasis of 96 patients with metastatic pulmonary adenocarcinoma by immunhistochemically evaluation with the monoclonal antibody 4D1/C2. Expression of CEACAM1 was shown in $81.3 \%$ (78 out of 96 ) primary tumors. Of the 76 patients with a CEACAM1 expressing primary tumor, $75(98.7 \%)$ had CEACAM1 expressing tumor cells in local lymph nodes. Of the 18 patients without CEACAM1 expression in the primary tumor, 13 $(72.2 \%)$ showed no CEACAM1 expression, whereas
$5(27.8 \%)$ patients did. In this study 9 hematogenous metastasis were also investigated and 7 metastasis $(77.8 \%)$ showed CEACAM1 expression of the tumor cells. In these cases, the primary tumor also showed CEACAM1 expression. In the two cases in which the metastasis showed no CEACAM1 expression, the primary tumor was also CEACAM1 negative. So, a significant positive correlation was found between CEACAM1 expression on cells of the primary tumor, lymph node metastasis $(\mathrm{p}<0.005)$ and hematogenous metastasis $(p=0.03)$. CEACAM1 expression did not correlate with stage, gender, grading or patient's age. Compared to patients with tumors not expressing CEACAM1, patients with a CEACAM1expressing tumor had a shorter median overall survival (21 vs 28 months) and progression-free survival (11.7 vs 16.3 months). In conclusion, the study conducted by Thöm et al. showed that CEACAM1 expression is preserved during lymphatic and hematogenous tumor spread of adenocarcinoma of the lung. These results support the prognostic relevance of the expression of CEACAM1 in pulmonary adenocarcinoma and might allow classification of patients into prognostic groups, which could be important for future application of targeted therapy.

\section{EXPRESSION OF CEACAM1 IN COLON CANCER}

Several reports have shown that CEACAM1 is over expressed at protein level in colorectal cancer and correlated with clinical stage. Recently, Ieda et al. [72] have deeply investigated the implication of CEACAM1 re-expression in colorectal cancer. The immunohistochemical analyses were conducted with CEACAM1 long (CEACAM1-L) or short (CEACAM1-S) cytoplasmic domain-specific policlonal antibodies on clinical samples from 164 patients with colorectal cancer. In more detail, the antibodies to the short cytoplasmic domain of CEACAM1 were generated in one mouse using a peptide of 12 amino acids whose sequence corresponded to the entire short cytoplasmic domain (KKHFGKTGSSGPLQ) with two lysines at the $\mathrm{N}$ terminus. To generate antibodies to the long form, a peptide corresponding to the last 16 residues in the C-terminal region of CEACAM1 long cytoplasmic domain with two lysines at the $\mathrm{N}$-terminal end (KK PSLTATEIIYSEVKKQ) was injected into a rabbit. The immunohistochemical study conducted by Ieda and co-workers clearly demonstrated that the expression of both CEACAM1-L and -S was weak at the luminal surface of advanced colorectal cancers and was more intense at the invasion front of advanced colorectal cancers with differences between the two cytoplasmic isoforms expression levels. In particular, the authors showed that CEACAM1-L dominance, in comparison with CEACAM1-S, at the invasion front of colorectal cancer is associated with lymph node involvement, hematogenous metastasis and shorter survival of patients, indicating that re-ex- 
pression of CEACAM1, in particular long cytoplasmic domain isoform, may function for invasion and migration of colorectal cancer cells.

\section{CONCLUSION}

The treatment of cancer showing an intrinsic or acquired chemical or immune resistance remains a formidable challenge owing to factors such as the difficulties in differentiating tumor cells from healthy cells to ameliorate the disease without causing intolerable toxicity to patients [73]. Finding new markers useful for the stratification of patients may offer a new prospective for the development of more efficacious therapies [74]. As reported and discussed in this article, de novo expression of CEACAM1 is associated with progression and reduced disease-free survival of melanoma, adenocarcinoma of the lung and other several solid tumors that are intrinsically resistant to chemotherapy or evolve in MDR variants under the selective pressure of cytotoxic drugs [69]. Therefore, CEACAM1 seems to be a good tumor marker for the stratification of risk in cancer patients. In addition, CEACAM1 has an important role in the progression, invasion and metastatic potential

\section{References}

1. Hammarstrom $S$. The carcinoembryonic antigen (CEA) family: structures, suggested functions and expression in normal and malignant tissues. Semin Cancer Biol 1999;9:67-81. http://dx.doi.org/10.1006/scbi.1998.0119

2. De Angelis M, Heinrich G, Dai T, Bowman TA, Patel PR, Lee SJ, Hong EG, Jung DY, Assmann A, Kulkarni, RN, Kim JK e Najjar S. Carcinoembryonic antigen-related cell adhesion molecule I. A link between insulin and lipid metabolism. Diabetes 2008;57:2296-303.

http://dx.doi.org/10.2337/db08-0379

3. Wagener C, Ergün S. Angiogenic properties of the carcinoembryonic antigen-related cell adhesion molecule 1. Exp Cell Res 2000;261(1):19-24. http://dx.doi.org/10.1006/excr.2000.5038

4. Gray-Owen SD, Blumberg RS. CEACAM1: contact-dependent control of immunity. Nat Rev Immunol 2006;6(6):433-46. http://dx.doi.org/10.1038/nri1864

5. Gray-Owen SD. Neisserial Opa proteins: impact on colonization, dissemination and immunity. Scand J Infect Dis 2003; 35(9):614-8. http://dx.doi.org/10.1080/00365540310016042

6. Hemmila E, Turbide C, Olson M, Jothy S, Holmes KV, Beauchemin N. Ceacamla-/- mice are completely resistant to infection by murine coronavirus mouse hepatitis virus A59. J Virol 2004;78(18):10156-65. http://dx.doi.org/10.1128/JVI.78.18.10156-10165.2004

7. Voges M, Bachmann V, Kammerer R, Gophna U, Hauck CR. CEACAM1 recognition by bacterial pathogens is species-specific. BMC Microbiol 2010;10:117-28. http://dx.doi.org/10.1186/1471-2180-10-117

8. Kuespert K, Pils S, Hauck CR. CEACAMs: their role in physiology and pathophysiology. Curr Opin Cell Biol 2006; 18:565-71.

http://dx.doi.org/10.1016/j.ceb.2006.08.008

9. Watt SM, Teixeira AM, Zhou GQ, Doyonnas R, Zhang Y, Grunert F, Blumberg RS, Kuroki M, Skubitz KM, Bates of cancer and is involved in the tumor angiogenesis. So, this adhesion molecule also has all the characteristics for a good molecular target. Monoclonal antibodies represent the fastest growing sector of pharmaceutical biotechnology and a number of antibody-based biopharmaceuticals have been approved for cancer treatment [15-17]. The results concerning CEACAM1 distribution, function and dysregulation indicate the importance of moving towards personalized medicine as it is well recognized that cancers classified based on traditional morphologic assessments are greatly heterogeneous [68]. In this context we have utilized most of the recent insights gained on the role of CEACAM1 as a tumor marker to design, in the near future, novel forms of in vivo immuno- therapeutic agents for large and possibly homogeneous sub-populations of patients.

\section{Conflict of interest statement}

There are no potential conflicts of interest or any financial or personal relationships with other people or organizations that could inappropriately bias conduct and findings of this study.

Received on 13 January 2012.

Accepted on 12 March 2012.
PA. Homophilic adhesion of human CEACAM1 involves N-terminal domain interactions: structural analysis of the binding site. Blood 2001;98:1469-79. http://dx.doi.org/10.1182/blood.V98.5.1469

10. Stern N, Markel G, Arnon TI, Gruda R, Wong H, GrayOwen SD, Mandelboim O. Carcinoembryonic antigen (CEA) inhibits NK killing via interaction with CEA-related cell adhesion molecule 1. J Immunol 2005;174:6692-701.

11. Chen T, Zimmermann W, Parker J, Chen I, Maeda A, Bolland S. Biliary glycoprotein (BGPa, CD66a, CEACAM1) mediates inhibitory signals. J Leukocyte Biol 2001;70:335-40.

12. Kammerer R, Riesenberg R, Weiler C, Lohrmann J, Schleypen J, Zimmermann W. The tumor suppressor gene CEACAM1 is completely but reversibly downregulated in renal cell carcinoma. J Pathol 2004;204(3):258-67. http://dx.doi.org/10.1002/path.1657

13. Thies A, Moll I, Berger J, Wagener C, Brummer J, Schulze HJ, Brunner G, Schumacher U. CEACAM-1 expression in cutaneous malignant melanoma predicts the development of metastatic disease. J Clin Oncol 2002;20:2530-6. http://dx.doi.org/10.1200/JCO.2002.05.033

14. Laack E, Nikbakht H, Peters A, Kugler C, Jasiewicz Y, Edler L, Brummer J, Schumacher U, Hossfeld DK. Expression of CEACAM-1 in adenocarcinoma of the lung: a factor of independent prognostic significance. J Clin Oncol 2002;20: 4279-84. http://dx.doi.org/10.1200/JCO.2002.08.067

15. Reichert JM, Rosensweig CJ, Faden LB, Dewitz MC. Monoclonal antibody successes in the clinic. Nat Biotechnol 2005; 23(9):1073-8. http://dx.doi.org/10.1038/nbt0905-1073

16. Carter P. Improving the efficacy of antibody-based cancer therapies. Nature Rev Cancer 2001;118:29. http://dx.doi.org/10.1038/35101072

17. Payne G. Progress in immunoconjugate cancer therapeutics. Cancer Cell 2003;3:207-12. http://dx.doi.org/10.1016/S1535-6108(03)00057-6 
18. Schrama D, Reisfeld RA, Becker JC. Antibody targeted drugs as cancer therapeutics. Nature Rev Drug Discov 2006;5:147-59. http://dx.doi.org/10.1038/nrd1957

19. Senter PD. Potent antibody drug conjugates for cancer therapy. Curr Opin Chem Biol 2009;13:235-44. http://dx.doi.org/10.1016/j.cbpa.2009.03.023

20. Neumaier M, Paululat S, Chan A, Matthaes P, Wagener C. Biliary glycoprotein, a potential human cell adhesion molecule, is down-regulated in colorectal carcinomas. Proc Natl Acad Sci USA 1993;90:10744-8. http://dx.doi.org/10.1073/pnas.90.22.10744

21. Bamberger AM, Riethdorf L, Nollau P, Naumann M, Erdmann I, Gotze J, Brummer J, Schulte H M, Wagener C, Loning T. Dysregulated expression of CD66a (BGP. C-CAM), an adhesion molecule of the CEA family, in endometrial cancer. Am J Pathol 1998;152:1401-6.

22. Riethdorf L, Liboa BW, Henkel U, Naumann M, Wagener C, Löning T. Differential expression of CD66a (BGP), a cell adhesion molecule of the carcinoembryonic antigen family, in benign, premalignant, and malignant lesions of the human mammary gland. J Histochem Cytochem 1997;45:957-63. http://dx.doi.org/10.1177/002215549704500705

23. Lin $\mathrm{SH}, \mathrm{Pu}$ YS. Function and therapeutic implication of CCAM cell-adhesion molecule in prostate cancer. Semin Oncol 1999;26(2):227-33

24. Luo W, Tapolsky M, Earley K, Wood CG, Wilson DR, Logothetis CJ, Lin SH. Tumor-suppressive activity of CD66a in prostate cancer. Cancer Gene Ther 1999;6:313-21.

25. Thies A, Berlin A, Brunner G, Schulze HJ, Moll I, Pfüller U, Wagener C, Schachner M, Altevogt P, Schumacher U. Glycoconjugate profiling of primary melanoma and its sentinel node and distant metastases: implications for diagnosis and pathophysiology of metastases. Cancer Lett 2007; 248(1):68-80. http://dx.doi.org/10.1016/j.canlet.2006.05.020

26. Thies A, Pfüller U, Schachner M, Horny HP, Moll I, Schumacher U. Binding of mistletoe lectins to cutaneous malignant melanoma: Implications for prognosis and therapy. Anticancer Res 2001;21:2883-7.

27. Thies A, Moll I, Berger J, Schumacher U. Lectin binding to cutaneous malignant melanoma: HPA is associated with metastasis formation. Br J Cancer 2001;84:819-23. http://dx.doi.org/10.1054/bjoc.2000.1673

28. Gambichler T, Grothe S, Rotterdam S, Altmeyer P, Kreuter A. Protein expression of carcinoembryonic antigen cell adhesion molecules in benign and malignant melanocytic skin lesions. Am J Clin Pathol 2009;131(6):782-87. http://dx.doi.org/10.1309/AJCP24KXJVBZXENS

29. Markel G, Seidman R, Cohen Y, Besser MJ, Sinai TC, Treves AJ, Orenstein A, Berger R, Schachter J. Dynamic expression of protective CEACAM1 on melanoma cells during specific immune attack. Immunology 2009;126(2):186-200. http://dx.doi.org/10.1111/j.1365-2567.2008.02888.x

30. Markel G, Lieberman N, Katz G, Arnon TI, Lotem M, Drize O, Blumberg RS, Bar-Haim E, Mader R, Eisenbach L, Mandelboim O. CD66 interactions between human melanoma and NK cells: a novel class I MHC-independent inhibitory mechanism of cytotoxicity. J Immunol 2002;168:2803-10.

31. Markel G, Wolf D, Hanna J Gazit R, Goldman-Wohl D, Lavy Y, Yagel S, Mandelboim O. Pivotal role of CEACAM1 protein in the inhibition of activated decidual lymphocyte functions. $J$ Clin Invest 2002;110:943-53.

http://dx.doi.org/10.1172/JCI200215643

32. Markel G, Seidman R, Stern N Cohen-Sinai T, Izhaki O, Katz G, Besser M, Treves AJ, Blumberg RS, Loewenthal R, Mandelboim O, Orenstein A, Schachter J. Inhibition of human tumor-inWltrating lymphocyte eVector functions by the hemophilic carcinoembryonic cell adhesion molecule 1 interactions. J Immunol 2006;177:6062-71.

33. Sanders DS, Evans AT, Allen CA, Bryant FJ, Johnson GD, Hopkins J, Stocks SC, Marsden JR, Kerr MA. Classification of CEA-related positivity in primary and metastatic malignant melanoma. J Pathol 1994;172(4):343-8. http://dx.doi.org/10.1002/path.1711720409

34. Ravindranath MH, Shen P, Habal N, Soh D, Nishimoto K, Gonzales A, Tam WY, Bilchik A, Morton DL. Does human melanoma express carcinoembryonic antigen? Anticancer Res 2000;20(5A):3083-92.

35. Selby WL, Nance KV, Park HK. CEA immunoreactivity in metastatic malignant melanoma. Mod Pathol 1992;5(4):415-9.

36. Kim CJ, Reintgen DS, Balch CM; AJCC Melanoma Staging Committee. The new melanoma staging system. Cancer Control 2002;9(1):9-15.

37. Balch CM, Buzaid AC, Soong SJ, Atkins MB, Cascinelli N, Coit DG, Fleming ID, Gershenwald JE, Houghton AJr, Kirkwood JM, McMasters KM, Mihm MF, Morton DL, Reintgen DS, Ross MI, Sober A, Thompson JA, Thompson JF. Final version of the American Joint Committee on Cancer staging system for cutaneous melanoma. J Clin Oncol 2001;19:3635-48.

38. Blaheta HJ, Schittek B, Breuninger H, Garbe C. Detection of micrometastasis in sentinel lymph nodes of patients with primary cutaneous melanoma. Recent Results Cancer Res 2001; 158:137-46. http://dx.doi.org/10.1007/978-3-642-59537-0_14

39. Karimipour DJ, Lowe L, Su L, Hamilton T, Sondak V, Johnson TM, Fullen D. Standard immunostains for melanoma in sentinel lymph node specimens: which ones are most useful? J Am Acad Dermatol 2004;50(5):759-64. http://dx.doi.org/10.1016/j.jaad.2003.07.016

40. Kucher C, Zhang PJ, Acs G, Roberts S, Xu X. Can MelanA replace S-100 and HMB-45 in the evaluation of sentinel lymph nodes from patients with malignant melanoma?. Appl Immunohistochem Mol Morphol 2006;14(3):324-7. http://dx.doi.org/10.1097/00129039-200609000-00011

41. Okegawa T, Pong RC, Li Y, Hsieh JT. The role of cell adhesion molecule in cancer progression and its application in cancer therapy. Acta Biochim Pol 2004;51:445-57.

42. Brooks SA. The involvement of Helix pomatia lectin (HPA) binding $\mathrm{N}$-acetylgalactosamine glycans in cancer progression. Histol Histopathol 2000;15(1):143-58.

43. Pryme IF, Bardocz S, Pusztai A, Ewen SW. Suppression of growth of tumour cell lines in vitro and tumours in vivo by mistletoe lectins. Histol Histopathol 2006;21(3):285-99.

44. Szász F, Sirivaidyapong S, Cheng FP, Voorhout WF, Marks A, Colenbrander B, Solti And L, Gadella BM. Detection of calcium ionophore induced membrane changes in dog sperm as a simple method to predict the cryopreservability of dog semen. Mol Reprod Dev 2000; 55(3):289-98. http://dx.doi.org/10.1002/(SICI)1098-2795(200003)55:3<289:: AID-MRD7>3.0.CO;2-K

45. Avilés M, Castells MT, Martínez-Menárguez JA, Abascal I, Ballesta J. Localization of penultimate carbohydrate residues in zona pellucida and acrosomes by means of lectin cytochemistry and enzymatic treatments. Histochem J 1997; 29(8):583-92.

46. Chen L, Ko CP. Extension of synaptic extracellular matrix during nerve terminal sprouting in living frog neuromuscular junctions. J Neurosci 1994;14(2):796-808.

47. Cochran AJ, Wen DR, Berthier-Vergnes O, Bailly C, Doré JF, Bérard F, Moulin G, Thomas L. Cytoplasmic accumulation of peanut agglutinin-binding glycoconjugates in the cells of primary melanoma correlates with clinical outcome. Hum Pathol 1999;30(5):556-61. http://dx.doi.org/10.1016/S0046-8177(99)90201-0 
48. Ota T, Kohno H, Maeda M, Tanino M, Odashima S. Involvement of peanut agglutinin-binding sugar chains in experimental metastasis of B16 melanoma cells. Oncol Res 1993; 5(6-7):235-43.

49. Carmeliet P. Manipulating angiogenesis in medicine. J Intern Med 2004;255:538-61. http://dx.doi.org/10.1111/j.1365-2796.2003.01297.x

50. Folkman J. Fundamental concepts of the angiogenic process. Curr Mol Med 2003;3:643-51. http://dx.doi.org/10.2174/1566524033479465

51. Dejana E, Spagnuolo R, Bazzoni G. Interendothelial junctions and their role in the control of angiogenesis, vascular permeability and leukocyte transmigration. Thromb Haemost 2001;86:308-15.

52. Ergun S, Kilik N, Ziegeler G, Hansen A, Nollau P, Gotze J, Wurmbach J H, Horst A, Weil J, Fernando M, Wagener C. CEA-related cell adhesion molecule 1: a potent angiogenic factor and a major effector of vascular endothelial growth factor. Mol Cell 2000;5:311-20.

http://dx.doi.org/10.1016/S1097-2765(00)80426-8

53. Kilic N, Oliveira-Ferrer L, Wurmbach JH, Loges S, Chalajour F, Neshat-Vahid S, Weil J, Fernando M, Ergun $\mathrm{S}$. Pro-angiogenic signaling by the endothelial presence of CEACAM1. J Biol Chem 2005;280:2361-9. http://dx.doi.org/10.1074/jbc.M409407200

54. Oliveira-Ferrer L, Tilki D, Ziegeler G, Hauschild J, Loges S, Irmak S, Kilic E, Huland H, Friedrich M, Ergün S. Dual role of carcinoembryonic antigen-related cell adhesion molecule 1 in angiogenesis and invasion of human urinary bladder cancer. Cancer Res 2004;64:8932-8. http://dx.doi.org/10.1158/0008-5472.CAN-04-0505

55. Tilki D, Irmak S, Oliveira-Ferrer L, Hauschild J, Miethe K, Atakaya H, Hammerer P, Friedrich MG, Schuch G, Galalae R, Stief CG, Kilic E, Huland H, Ergun S.CEA-related cell adhesion molecule-1 is involved in angiogenic switch in prostate cancer. Oncogene 2006;25:4965-74. http://dx.doi.org/10.1038/sj.onc.1209514

56. Dango S, Sienel W, Schreiber M, Stremmel C, Kirschbaum A, Pantel K, Passlick B. Elevated expression of carcinoembryonic antigen-related cell adhesion molecule 1 (CEACAM1 ) is associated with increased angiogenic potential in nonsmall-cell lung cancer. Lung Cancer 2008;60(3):426-33. http://dx.doi.org/10.1016/j.lungcan.2007.11.015

57. Liu W, Wei W, Winer D, Bamberger A-M, Bamberger C, Wagener C, Ezzat S, Asa SL. CEACAM1 impedes thyroid cancer growth but promotes invasiveness: a putative mechanism for early metastases. Oncogene 2007;26:2747-58. http://dx.doi.org/10.1038/sj.onc.1210077

58. Dupont WD, ParlFF, Hartmann WH, Brinton LA, Winfield AC, WorrellJA,SchuylerPA,PlummerWD. Breastcancerriskassociated with proliferativebreastdiseaseandatypicalhyperplasia. Cancer 1993;71:1258-65

http://dx.doi.org/10.1002/1097-0142(19930215)71:4<1258:: AID-CNCR2820710415>3.0.CO;2-I

59. Ouyang XS, Wang X, Ling MT, Wong HL, Tsao SW, Wong YC. Id-1 stimulates serum independent prostate cancer cell proliferation through inactivation of $\mathrm{p} 16(\mathrm{INK} 4 \mathrm{a}) / \mathrm{pRB}$ pathway. Carcinogenesis 2002;23:721-5. http://dx.doi.org/10.1093/carcin/23.5.721

60. Singh J, Murata K, Itahana Y, Desprez PY. Constitutive expression of the Id-1 promoter in human metastatic breast cancer cells is linked with the loss of NF-1/Rb/HDAC-1 transcription repressor complex. Oncogene 2002;21(12):1812-22. http://dx.doi.org/10.1038/sj.onc.1205252

61. Schoppmann SF, Schindl M, Bayer G, Aumayr K, Dienes J, Horvat R, Rudas M, Gnant M, Jakesz R, Birner P.
Overexpression of Id-1 is associated with poor clinical outcome in node negative breast cancer. Int J Cancer 2003;104: 677-82.

http://dx.doi.org/10.1002/ijc.11009

62. Liu Q, Yang YM, Zhang QH, Zhang TG, Zhou Q, Zhou CJ. Inhibitor of differentiation is overexpressed with progression of benigntomalignantlesionsand related with CEACAM1 distribution in mammary glands. Ann Diagn Pathol 2011;15(1):30-6. http://dx.doi.org/10.1016/j.anndiagpath.2010.07.010

63. Zhou CJ, Liu B, Zhu KX, Zhang QH, Zhang TG, Xu WH, Wang HB, Yu WH, Qu YD, Wang HJ, Wu HL, Sun SZ, Guo JQ. The different expression of carcinoembryonic antigen-related cell adhesion molecule 1 (CEACAM1) and possible roles in gastric carcinomas. Pathol Res Pract 2009;205(7):483-9. http://dx.doi.org/10.1016/j.prp.2009.01.006

64. Cavallaro U, Christofori G. Cell adhesion in tumor invasion and metastasis: loss of the glue is not enough. Biochim Biophys Acta 2001;1552:39-45. http://dx.doi.org/10.1016/S0304-419X(01)00038-5

65. Pignatelli M, Vessey C J. Adhesion molecules: novel molecular tools in tumor pathology. Hum Pathol 1994;25:849-56. http://dx.doi.org/10.1016/0046-8177(94)90002-7

66. Brümmer J, Ebrahimnejad A, Flayeh R, Schumacher U, Löning T, Bamberger AM, Wagener C. Cis Interaction of the cell adhesion molecule CEACAM1 with integrin beta(3) Am J Pathol 2001;159(2):537-46 http://dx.doi.org/10.1016/S0002-9440(10)61725-7

67. Howe HL, Wingo PA, Thun MJ, Ries LA, Rosenberg HM, Feigal EG, Edwards BK. Annual report to the nation on the status of cancer (1973 through 1998), featuring cancers with recent increasing trends. J Natl Cancer Inst 2001;93:824-42. http://dx.doi.org/10.1093/jnci/93.11.824

68. Torky M, el Zeky F, Hall JC. Significant changes in the distribution of histologic types of lung cancer. A review of 4928 cases. Cancer 1990;65:2361-7.

http://dx.doi.org/10.1002/1097-0142(19900515)65:10<2361:: AID-CNCR2820651034>3.0.CO;2-C

69. Sienel W, Dango S, Woelfle U, Morresi-Hauf A, Wagener C, Brümmer J, Mutschler W, Passlick B, Pantel K. Elevated expression of carcinoembryonic antigen-related cell adhesion molecule 1 promotes progression of non-small cell lung cancer. Clin Cancer Res 2003;9(6):2260-6.

70. Liu NS, Spitz MR, Kemp BL, Cooksley C, Fossella FV, Lee JS, Hong WK, Khuri FR. Adenocarcinoma of the lung in young patients: The M. D. Anderson experience. Cancer 2000; $88: 1837-41$.

h t t p://dx.doi.org/10.1002/( S I C I ) 1097 0142(20000415)88:8<1837::AID-CNCR12>3.0.CO;2-E

71. Thöm I, Schult-Kronefeld O, Burkholder I, Schuch G, Andritzky B, Kastendieck H, Edler L, Wagener C, Bokemeyer C, Schumacher U, Laack E. Expression of CEACAM-1 in pulmonary adenocarcinomas and their metastases. Anticancer Res 2009;9(1):249-54.

72. Ieda J, Yokoyama S, Tamura K, Takifuji K, Hotta T, Matsuda K, Oku Y, Nasu T, Kiriyama S, Yamamoto N, Nakamura Y, Shively JE, Yamaue H. Re-expression of CEACAM1 long cytoplasmic domain isoform is associated with invasion and migration of colorectal cancer. Int $J$ Cancer 2011;129(6):1351-61. http://dx.doi.org/10.1002/ijc. 26072

73. Scagliotti GV, Novello S, Selvaggi G. Multidrug resistance in non-small- lung cancer. Ann Oncol 1999;10(Suppl. 5):S83-6. http://dx.doi.org/10.1093/annonc/10.suppl_5.S83

74. Gottesman MM, Fojo T, Bates SE: Multidrug resistance in cancer: role of ATP-dependent transporters. Nat Rev Cancer 2002;2:48-58.

http://dx.doi.org/10.1038/nrc706 